\title{
Diseño de directrices para el tablero de un puente curvo atirantado, mediante curvas funiculares y antifuniculares de la carga concéntrica introducida por los tirantes
}

\section{Design of a curved cable-stayed bridge deck's directives, by the funicular and antifunicular curves of the concentric load introduced by the cables}

D. Galante Bardín $^{(*)}$, M.L.A. Astiz Suárez ${ }^{(*)}$

\section{RESUMEN}

El presente trabajo trata de resolver la optimización del trazado del tablero para un puente en curva. La directriz que se propone pretende conseguir que todo el tablero quede exento de flexiones de eje vertical. Para este diseño se plantean dos formulaciones diferentes, una discreta mediante sumatorios y otra continua mediante ecuaciones diferenciales no lineales. En ambas se estudian los dos posibles signos de la solicitación axil que va a dominar el diseño. Para facilitar la implantación de estas formulaciones, se propone una aproximación mediante mínimos cuadrados. El trabajo de completa con dos ejemplos de aplicación.

Palabras clave: Puente curvo atirantado; tablero funicular; tablero antifunicular; carga concéntrica; solicitación axil.

\section{ABSTRACT}

The aim of the following study is to optimize the layout of a curved bridge deck. The guideline proposed is intended to ensure that the entire board is free of vertical axis bending moments. Two mathematical approaches are developed for the directive, a discrete and a continuous. For both, positive and negative sign for the axial load in the deck is studied. To make the possible implementation easier, a least square approximation is made for both signs. Two examples will be developed to end the article.

Keywords: Curved cable-stayed bridge; funicular deck; antifunicular deck; concentric load; axial load.

(*) Escuela Técnica Superior de Caminos, Canales y Puertos (UPM). (España) Persona de contacto/Corresponding author: dario.galante@idiingenieros.es (D. Galante Bardín) ORCID: http://orcid.org/oooo-0002-0793-6077 (D. Galante Bardín); http://orcid.org/oooo-0002-4070-215X (M.L A. Astiz Suárez)

Cómo citar este artículo/Citation: Galante Bardín, D., Astiz Suárez, M.L.A. (2017). Diseño de directrices para el tablero de un puente curvo atirantado, mediante curvas funiculares y antifuniculares de la carga concéntrica introducida por los tirantes. Informes de la Construcción, 69(548): e228, doi: http://dx.doi.org/10.3989/ic.16.094.

Copyright: (C) 2017 CSIC. Licencia / License: Salvo indicación contraria, todos los contenidos de la edición electrónica de Informes de la Construcción se distribuyen bajo una licencia de uso y distribución Creative Commons Attribution License (CC BY) Spain 3.o. 


\section{INTRODUCCIÓN}

El puente curvo permite resolver las cada vez más estrictas y complejas restricciones geométricas que poseen las vías actuales. La naturaleza de estas restricciones es muy diversa, desde una mezcla entre obstáculos naturales y una mayor concienciación ecológica, pasando por derechos de paso en zonas urbanas hasta el desarrollo de grandes cruces entre viales a diferentes alturas, con enlaces entre ellos. Hasta no hace demasiado tiempo, la tipología de puente curvo utilizada era, casi de manera exclusiva, la de tablero sobre apoyos puntuales. Es esta tipología la que más se ha desarrollado por parte de investigadores (1) y normativas (2). En el caso de que existan restricciones que impidan esta tipología, lo habitual es la adaptación del entorno, para eliminar estas restricciones o conseguir la validez de alguna tipología de puente recto. La falta de experiencia, de suficiente documentación técnica, de cobertura por parte de normativas, de metodologías de diseño claras, sumado a las dudas que ofrecen los complejos métodos constructivos hasta ahora aplicados, disuaden a los ingenieros de diseños de puentes curvos más complejos. En los últimos años, gracias a las nuevas tecnologías (3) (4), se ha podido ver una cierta evolución en diferentes tipologías de puentes curvos, como puentes atirantados, colgantes o en arco. Este progreso se ha centrado fundamentalmente en el uso peatonal, donde ingenieros como Schlaich han desarrollado sistemas muy acertados con puentes colgantes y arco (5) o trabajos científicos con arcos adaptados a las cargas como los realizados por Lorenz Lachauer y Toni Kotnik (6) o Leonardo Todisco (7).

La acción axil presenta multitud de beneficios sobre las acciones transversales, en particular beneficios frente al momento flector de eje vertical (la relación sobre la que se va a desarrollar el artículo). Desde un punto de vista resistivo, la independencia existente entre la solicitación axil, de las formas funiculares y anti-funiculares (8), y la luz cubierta por el tablero convierte a la solicitación axil en la única posibilidad realista para soportar las cargas horizontales, introducidas por los tirantes al soportar las cargas verticales, en el plano del tablero (9). Desde el punto de vista de rigidez transversal del tablero (frente a la carga concéntrica introducida por los tirantes) y con ello de su correcto comportamiento en servicio. La flexibilidad del tablero frente a la solicitación axil es una función lineal de la luz del tablero, mientras que la combinación de flexibilidades del mismo frente a las solicitaciones de cortante en el plano de la curva y de momento transversal con eje vertical, son la suma de una función lineal (cortante) y una función cúbica de la luz del tablero (10).

Uno de los objetivos fundamentales del puente atirantado recto es conseguir un equilibrio transversal de las fuerzas de los tirantes (11), tanto en la cabeza de la torre como en el tablero. Este equilibrio no resulta complicado de conseguir, gracias a la combinación entre su bidimensionalidad y su triangulación (11). En los puentes atirantados curvos, se deshace la bidimensionalidad, acoplándose momento torsor, momento flector de eje radial, axil, cortante transversal y momento flector de eje vertical (12). Esto dificulta el equilibrio transversal entre las fuerzas de tracción de los tirantes, lo que provoca acciones sobre el tablero y la torre que fácilmente pueden llegar a ser determinantes en su dimensionamiento (13) (14). Que la resultante en el plano del tablero de estas cargas se resuelva mediante reacciones en el estribo tangenciales a la directriz, introduciendo exclusivamente solicitación axil sobre el tablero, depende de un correcto diseño del conjunto tablero, torre y tirantes. Un claro ejemplo de optimización de un sistema, con una carga concéntrica como carga principal, mediante el uso del diseño lo tenemos en la rueda de bicicleta, donde la curvatura de la llanta transforma la carga transversal de los radios en carga axil, hasta que finalmente la resultante se anula al cerrar la directriz de la llanta (15).

Basados en el diseño de la rueda de bicicleta, se han realizado puentes atirantados curvos posicionando la torre cerca del centro del arco desarrollado por el tablero (16). Este diseño conlleva 2 inconvenientes principales. Solo es viable con radios de arco muy pequeños (si no la distancia entre torre y tablero se hace excesiva). La torre no se posiciona en el centro de masas del tablero (suponiendo que estamos en el caso habitual de un arco de circunferencia), lo cual supone una componente horizontal en cabeza de torre que deberemos anclar de alguna manera (17) (18). Para resolver estos inconvenientes, algunos ingenieros han evolucionado el diseño de la torre y los tirantes para conseguir transmitir la misma acción transversal (que situando la torre en el centro de la circunferencia) en el plano del arco del tablero, sin tener que posicionar la torre en el centro de este arco. A la cabeza de este desarrollo podemos situar a Schlaich con sus pasarelas colgantes (5), donde las péndolas se distribuyen radialmente y se recogen mediante un cable portante. Mediante un correcto anclaje en los extremos, el cable portante lleva la carga a la cabeza de la torre, que se puede posicionar con mayor libertad. El eje de simetría radial, permite un equilibrio natural entre los cables portantes.

En este trabajo se va a cambiar el punto de inicio del que se ha partido habitualmente a la hora de afrontar un puente curvo atirantado. La tipología de la curvatura desarrollada por el tablero no va a ser un punto de partida, sino una consecuencia a partir de las necesidades y restricciones que tiene el puente. Mediante esta curvatura se va a buscar el equilibrio de las solicitaciones axiles frente a la carga de peso propio de la estructura (habitualmente la solicitación determinante en puentes de esta tipología), sin tener en cuenta las rigideces de cada elemento. Esta última omisión, se basa en el principio de que los tirantes se van a poner en carga para soportar el peso propio de la estructura, pudiendo incluir el resto de cargas muertas, sabiendo de antemano la carga vertical que va a sustentar cada uno de ellos (19) (20). En fases posteriores si se debe analizar el comportamiento del sistema frente a otras posibles cargas, donde la rigidez de los diferentes elementos del sistema, sí se tenga que tener en cuenta. La resultante de cada tirante sobre el tablero se hace pasar por el centro de cortante y masas, que se supone coincidente. Esto último puede no ser lo óptimo para posteriores solicitaciones torsoras sobre el tablero (21), pero ese análisis queda fuera del alcance de este artículo

\section{PRINCIPIOS DEL DISEÑO}

El denominado «Tablero AXIL», va a ser un tablero diseñado para funcionar fundamentalmente bajo la solicitación axil, sin que esto signifique que los tirantes que sustentan el tablero tengan que ser radiales. La directriz del tablero la va a marcar la dirección de la solicitación axil y a su vez la solicitación axil y su dirección la va a marcar la disposición y características de la torre (altura de la torre, y posición en planta de esta). La directriz, puede seguir la forma anti-funicular (axil de compresión) o funicular (axil de tracción) de las cargas 
transversales introducidas por los tirantes. La forma antifunicular, será cóncava respecto a la torre, girando en torno a la torre, mientras que la forma funicular será convexa, con una asíntota oblicua.

Para conseguir una directriz continua, se ha desestimado tener en cuenta los cambios axiles puntuales producidos por los tirantes que sustentan el tablero. Se resuelve el problema como si todo el tablero estuviese conectado a la cabeza de la torre, similar al cálculo de una catenaria continua.

Para una comprensión más intuitiva de cómo se concreta el diseño de este tipo de tableros, se van a presentar 2 tipos de cálculos de la directriz del tablero, uno discreto y otro continuo.

\section{CÁlCULO DISCRETO DE DIRECTRIZ}

En este cálculo, se va a discretizar el tablero en segmentos rectos, concentrando su masa en los nudos. En cada nudo se componen las fuerzas, obteniendo el ángulo del segmento que lo une con el siguiente nudo. Es obvio, que cuantos más nudos se introduzcan en el cálculo discreto, más se va a aproximar a un cálculo continuo (se verá finalmente que la única forma de resolver el problema, debido a su inherente no linealidad, pasa por un cálculo numérico, en el que al igual que en el cálculo discreto, se prevé la situación del siguiente punto a partir de la información que disponemos del anterior) (22).

El problema discreto, se describe en la Figura 1.

Si analizamos el nudo «i» de la Figura 1:

Donde:

$\mathrm{N}_{\mathrm{i}-1}$ : Carga axil en el tablero previa al nudo.

$\alpha_{\mathrm{i}-1}$ : Ángulo de la carga axil previa al nudo.

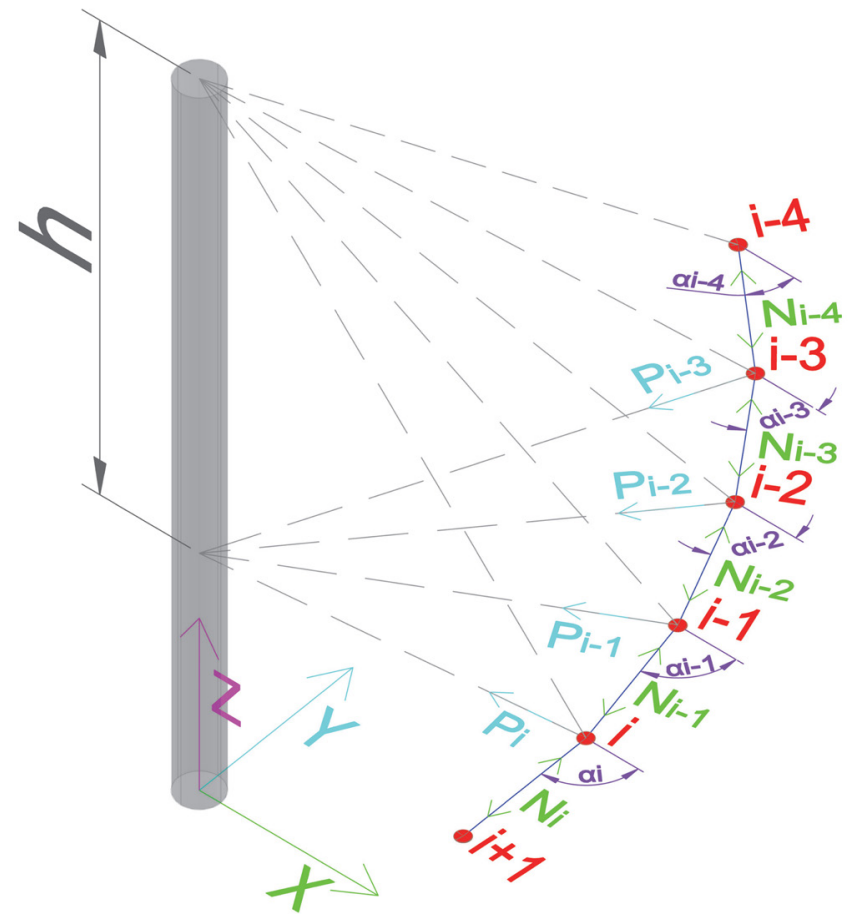

Figura 1. Cálculo discreto de directriz.
$\mathrm{P}_{\mathrm{i}}$ : Componente horizontal introducida por el tirante.

$\mathrm{P}_{\mathrm{Yi}} \mathrm{P}_{\mathrm{Xi}}$ : Componentes «Y» «X» de la carga horizontal introducida por el tirante que sustenta la masa.

$\mathrm{N}_{\mathrm{i}}$ : Carga axil en el tablero posterior al nudo.

$\alpha_{i}$ : Ángulo de la carga axil posterior al nudo.

Si componemos las cargas en el nudo, nos queda:

$$
\begin{aligned}
& \mathrm{N}_{\mathrm{i}-1} \cos \alpha_{\mathrm{i}-1}+\mathrm{P}_{\mathrm{Yi}}=\mathrm{N}_{\mathrm{i}} \cos \alpha_{\mathrm{i}} \\
& \mathrm{N}_{\mathrm{i}-1} \sin \alpha_{\mathrm{i}-1}+\mathrm{P}_{\mathrm{Xi}}=\mathrm{N}_{\mathrm{i}} \sin \alpha_{\mathrm{i}}
\end{aligned}
$$

Mientras que las cargas horizontales quedan:

$$
\begin{aligned}
& P_{Y i}=W_{z} \frac{y_{i}}{h} \\
& P_{X i}=W_{z} \frac{x_{i}}{h}
\end{aligned}
$$

Luego, a partir de unas condiciones de contorno conocidas (como una reacción en el estribo o la compresión o tracción en el cruce con el eje $\mathrm{x}-\mathrm{x}$ ), es fácil obtener la directriz «axil» del tablero discretamente a partir de sumatorios.

Por simplicidad de cálculo, en nuestro caso hemos supuesto que la directriz del tablero va a ser perpendicular al eje $\mathrm{x}$-x en el punto en que se cruza con este eje. Este punto se va a considerar una condición de contorno que denominaremos «Xo". También vamos a considerar como condición de contorno la reacción en este punto, reacción que vamos a denominar «FHT» (Fuerza axil en el tablero en el primer punto del desarrollo), cuya dirección va a coincidir con la de la directriz del tablero, perpendicular al eje $\mathrm{x}-\mathrm{x}$. Esta disposición, nos va a permitir desarrollar el tablero solo en un sentido, en nuestro caso hemos elegido el sentido «-y», sabiendo que el tablero va a ser simétrico respecto del eje $\mathrm{x}$-x. También por simplicidad se ha decidido posicionar la torre en el centro de coordenadas (Figura 2).

Con todas las condiciones de contorno descritas en el párrafo anterior, el problema discreto queda definido de la siguiente forma:

Los 2 primeros puntos coinciden en los 2 tipos de tableros.

\begin{tabular}{|c|}
\hline $\begin{array}{l}\text { Condiciones de contorno (2 primeros puntos). Tablero a } \\
\text { compresión y tablero a tracción [5] }\end{array}$ \\
\hline $\mathrm{x}[\mathrm{o}]=\mathrm{x}_{\mathrm{o}}$ \\
\hline $\mathrm{x}[1]=\mathrm{x}[\mathrm{o}]$ \\
\hline $\mathrm{y}[\mathrm{o}]=\mathrm{o}$ \\
\hline $\mathrm{y}[1]=-\frac{\mathrm{d}}{2}$ \\
\hline
\end{tabular}

Tablero a compresión:

Y para i $>2$ :

$$
x[i]=x[i-1]+d \frac{\frac{W z}{h p} \sum_{j=1}^{i-1} x[j]}{\sqrt{\left(\frac{W z}{h p} \sum_{j=1}^{i-1} x[j]\right)^{2}+\left(F H T+\frac{W z}{h p} \sum_{j=1}^{i-1} y[j]\right)^{2}}}
$$




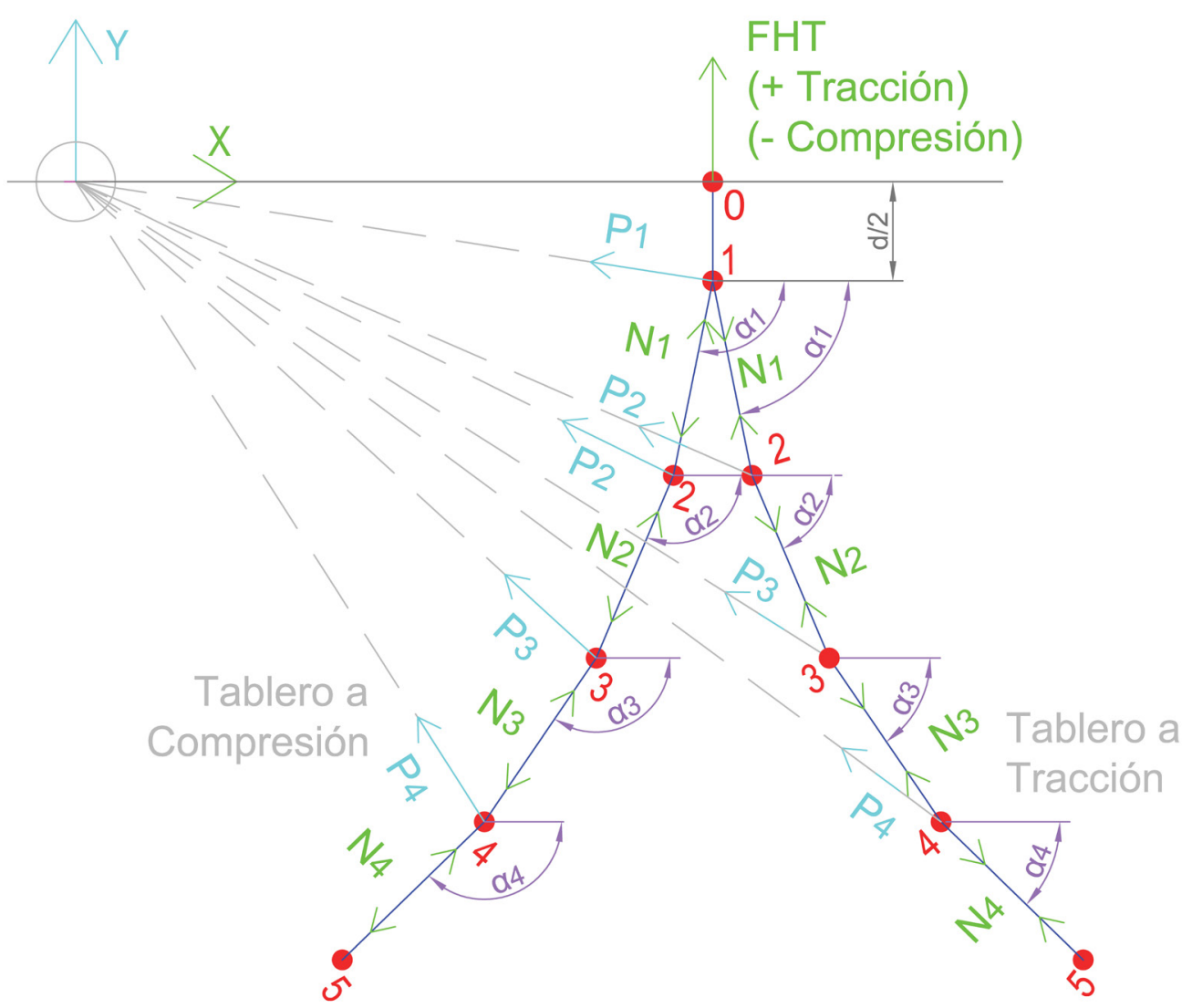

Figura 2. Cálculo discreto de directriz, condiciones de contorno. Funicular y antifunicular.

$$
y[i]=y[i-1]+d \frac{F H T+\frac{W z}{h p} \sum_{j=1}^{i-1} y[j]}{\sqrt{\left(\frac{W z}{h p} \sum_{j=1}^{i-1} x[j]\right)^{2}+\left(F H T+\frac{W z}{h p} \sum_{j=1}^{i-1} y[j]\right)^{2}}}
$$

Tablero a tracción:

Y para i $>2$ :

$$
\begin{aligned}
& x[i]=x[i-1]+d \frac{-\frac{W z}{h} \sum_{j=1}^{i-1} x[j]}{\sqrt{\left(-\frac{W z}{h p} \sum_{j=1}^{i-1} x[j]\right)^{2}+\left(-F H T-\frac{W z}{h p} \sum_{j=1}^{i-1} y[j]\right)^{2}}} \\
& y[i]=y[i-1]+d \frac{-F H T-\frac{W z}{h} \sum_{j=1}^{i-1} y[j]}{\sqrt{\left(-\frac{W z}{h p} \sum_{j=1}^{i-1} x[j]\right)^{2}+\left(-F H T-\frac{W z}{h p} \sum_{j=1}^{i-1} y[j]\right)^{2}}}
\end{aligned}
$$

Donde:

xo: Punto inicial del desarrollo, 1er corte con el eje $\mathrm{x}$-x (Condición de contorno).

FHT: Fuerza axil en el tablero en el primer punto del desarrollo (Positiva en el tablero a tracción y negativa en el tablero a compresión) (Condición de contorno).

d: Distancia entre nudos, minimizando esta distancia se aproxima el resultado del problema discreto al problema continuo (Condición de contorno).
Wz: Carga vertical sustentada por el tirante en cada nudo (Condición de contorno). En el caso de utilizar el peso propio como carga de diseño, sería el peso de un tramo «d» de tablero. hp: Altura de la torre sobre el tablero (Condición de contorno). $\mathrm{x}[\mathrm{i}-1]$ : Coordenada « $\mathrm{x} »$ del punto anterior. $y[\mathrm{i}-1]$ : Coordenada «y» del punto anterior.

$\mathrm{x}[\mathrm{i}]$ : Coordenada « $\mathrm{x}$ » del punto.

$y[i]$ : Coordenada «y» del punto

nt: $\mathrm{N}^{0}$ total de nudos que se van a estudiar, con la distancia «d» se puede calcular fácilmente la longitud del tablero (Condición de contorno).

Para finalizar el cálculo discreto, se van a presentar 2 ejemplos.

Condiciones de contorno. [10]

Compresión: Figura 3 y Figura 4.

Tracción: Figura 5 y Figura 6.

\begin{tabular}{|c|}
\hline $\mathrm{nt}=1000$ \\
\hline $\mathrm{hp}=50$ \\
\hline $\mathrm{Wz}=-1$ \\
\hline $\mathrm{x}_{\mathrm{o}}=60$ \\
\hline $\mathrm{d}=1$ \\
\hline Tablero a compresión: FHT $=-10$ \\
\hline Tablero a tracción: FHT $=50$ \\
\hline
\end{tabular}

En las Figuras 3 y 5, se puede ver el carácter opuesto que tienen las formas de los tableros que van a funcionar a tracción y a compresión. 


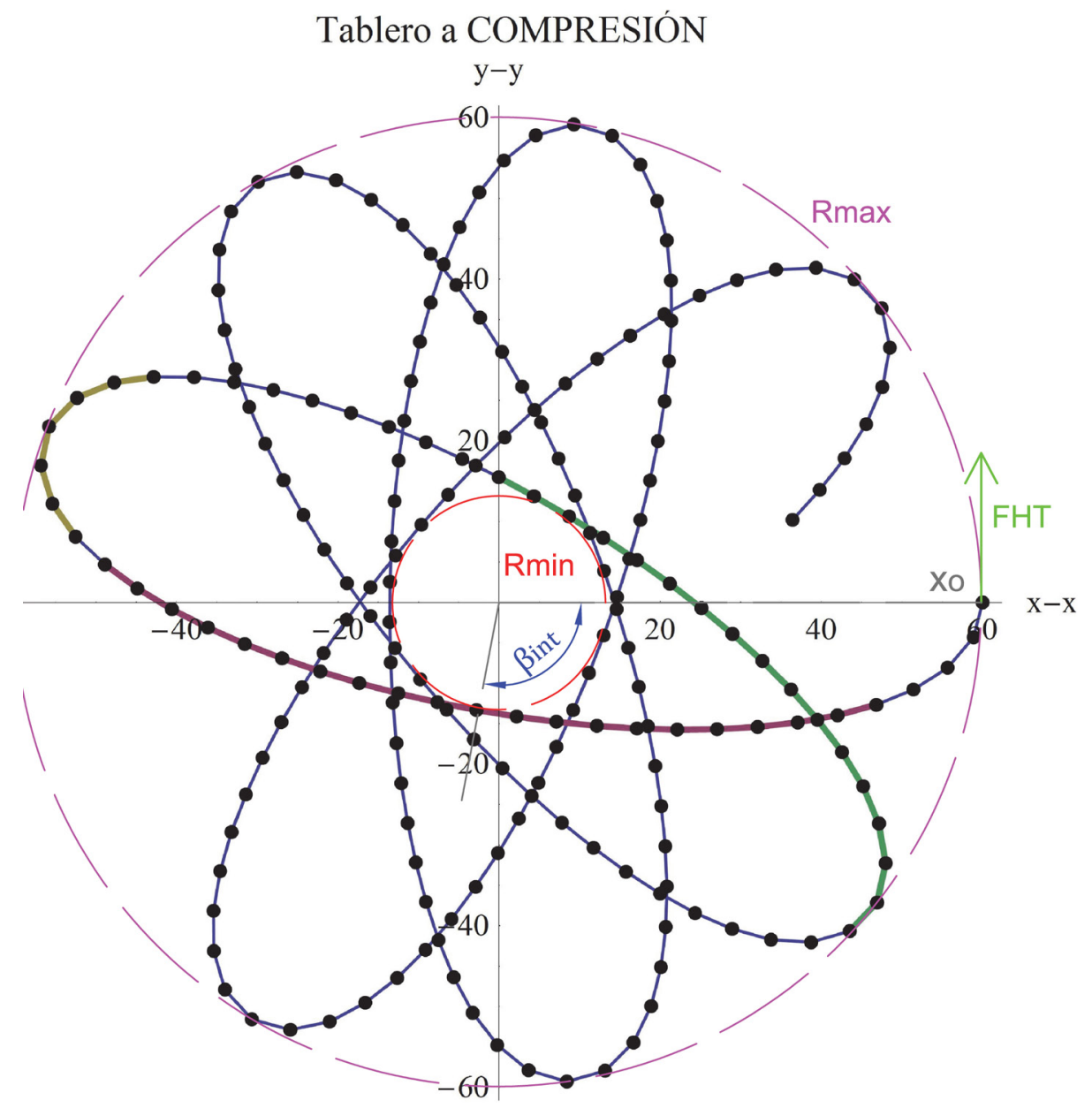

Figura 3. Directriz de tablero a compresión (antifunicular).

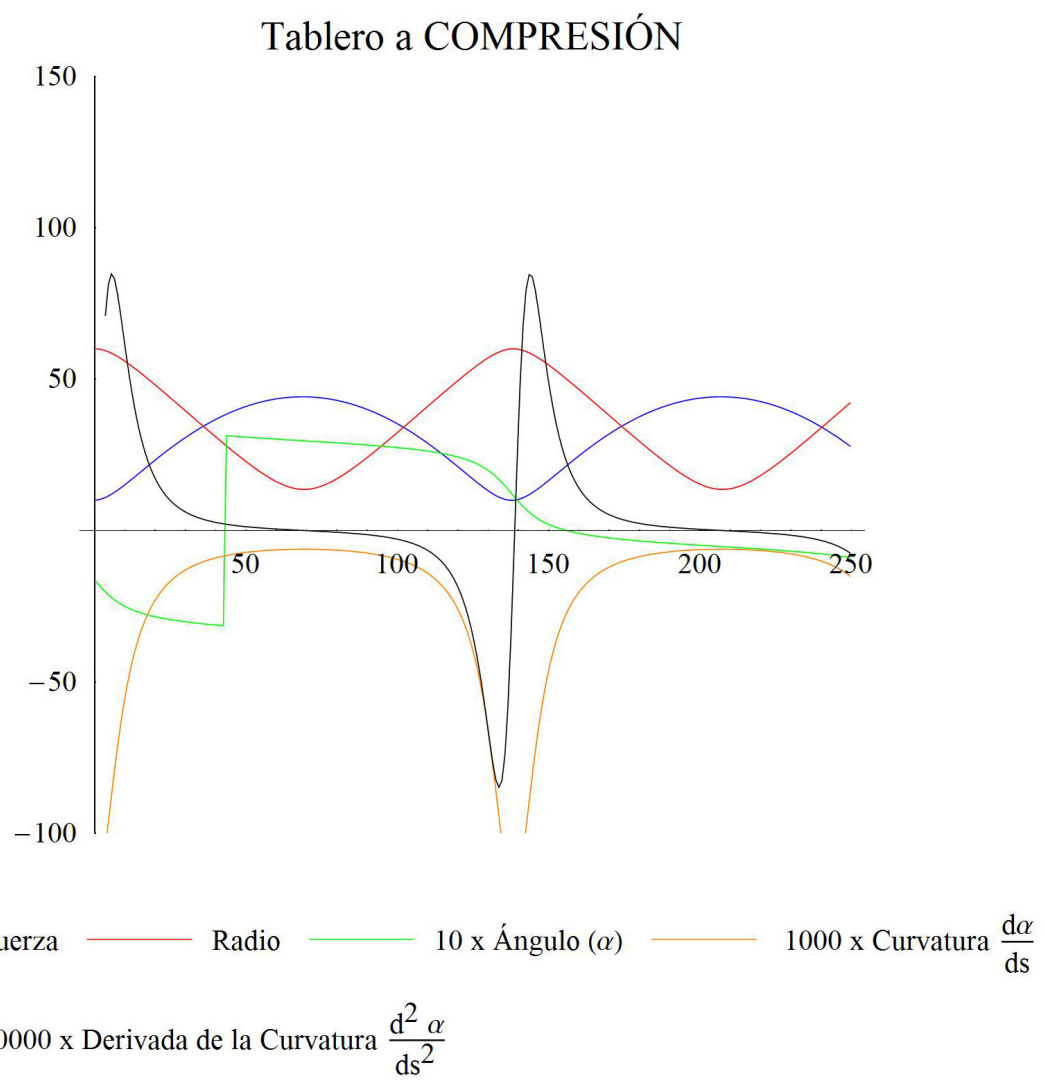

Figura 4. Variables, directriz de tablero a compresión (antifunicular). 


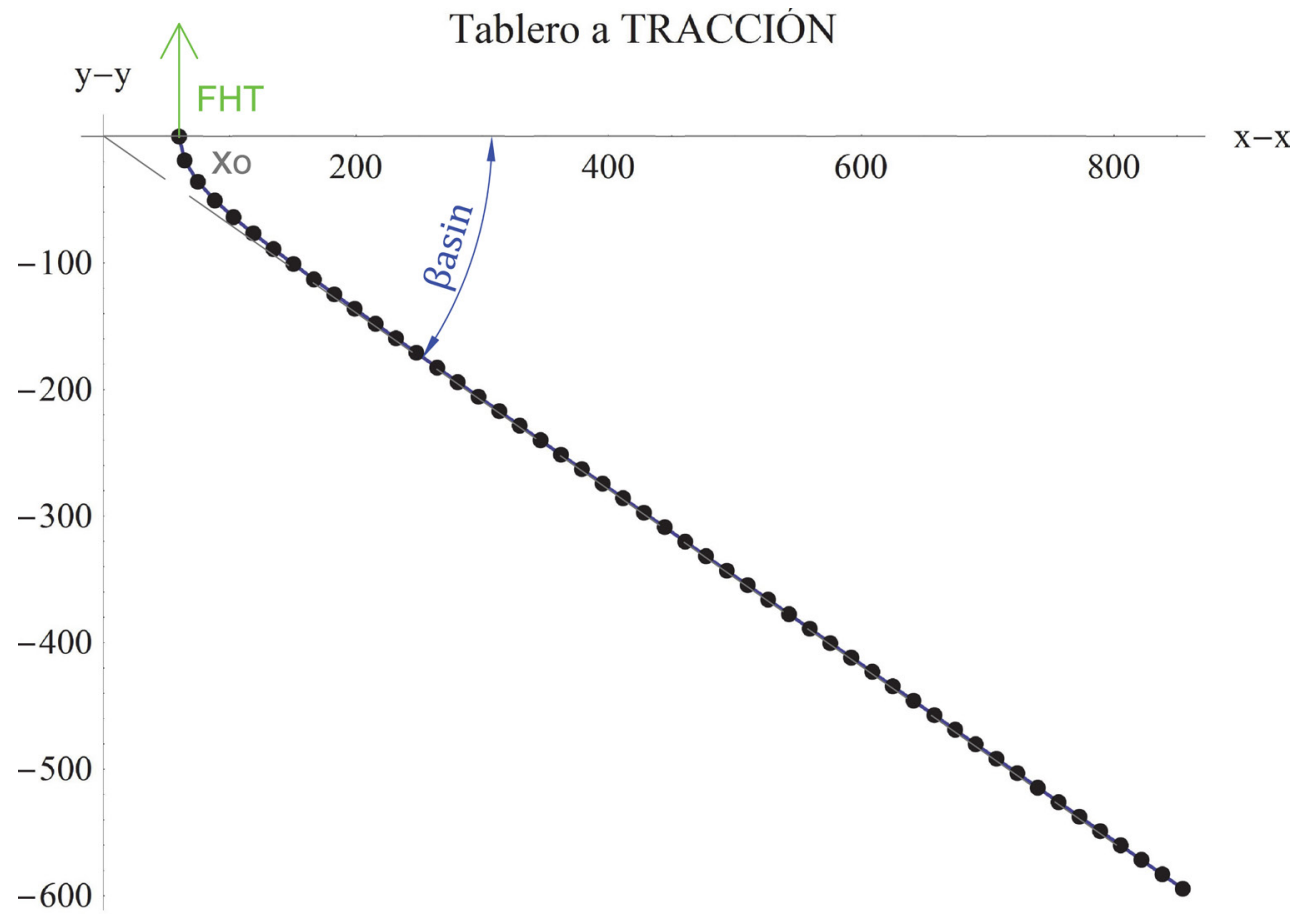

Figura 5. Directriz de tablero a tracción (funicular).

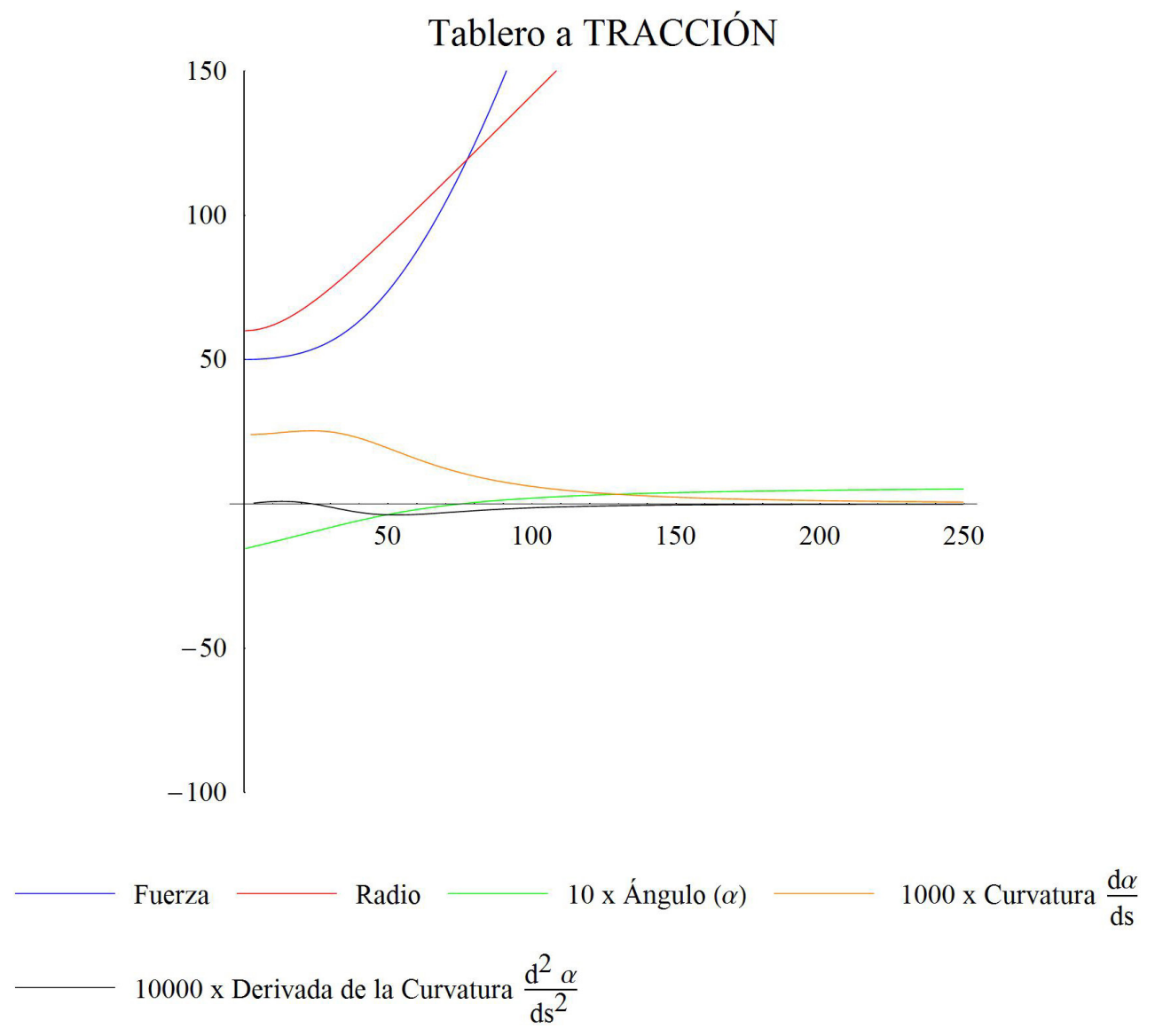

Figura 6. Variables, directriz de tablero a tracción (funicular).

En el caso del tablero trabajando a compresión (antifunicular), su directriz es cíclica (Figura 3), quedando su fuerza axil en el tablero y su radio, acotados entre unos determinados valores máximos y mínimos, cuando la perpendicular a la tangente pasa por la torre. Mientras que el ángulo es el que diverge. Ve- mos como el máximo radio, coincide con la menor solicitación axil y viceversa (Figura 4). A partir de ese punto las fuerzas exteriores invierten su relación con el axil del tablero, si es un punto de máxima de fuerza y mínimo radio, las cargas exteriores pasan de incrementar el axil a disminuirlo y viceversa. Se 
debe entender que la curva se ha desarrollado con las condiciones de contorno mostradas en la expresión [10], por comodidad de cálculo. Como directriz para el tablero, se puede coger cualquier tramo de la curva (en la Figura 3 se han escogido 3 posibles tramos). De la Figura 4 y Figura 6, se pueden obtener las nuevas condiciones de contorno que tendría cada tramo (acciones sobre los estribos, ángulo de tablero...).

En el caso del tablero trabajando a tracción (funicular), en su directriz se ve como la distancia respecto de la torre diverge según una asíntota oblicua (Figura 5). En este caso es el ángulo el que queda acotado y tiende a un valor estable, igualándose al ángulo de la asíntota oblicua, mientras que la fuerza y el radio son los que carecen de límite, tendiendo a infinito (Figura 6).

En las Figuras 4 y 6, se ha incluido la curvatura y su derivada. El objetivo es ver las posibilidades de desarrollar una curvatura compatible con el trazado de una carretera, compuesto por circunferencias y clotoides, que tienen estos tableros (23). Se puede apreciar como con las condiciones de contorno impuestas (al variar las condiciones varían las Figuras 4 y 6), sería sencillo escalonar la derivada de la curvatura, para ir encadenando clotoides (derivada de la curvatura: cte) y circunferencia (derivada de la curvatura: o) sin que la directriz sufriese grandes cambios. El desarrollo de estas aproximaciones excede el alcance de este trabajo.

\section{CÁlCULO CONTINUO DE LA DIRECTRIZ}

\subsection{Planteamiento del problema}

Para plantear el problema de forma continua, con la intención de obtener una solución analítica que resuelva la direc- triz del tablero, vamos a empezar por un diferencial de tablero (Figura 7).

$$
\begin{gathered}
\frac{d x}{d s}=\frac{N_{x}}{N} \\
\frac{d y}{d s}=\frac{N_{y}}{N} \\
\frac{N_{x}}{N_{y}}=\frac{d x}{d y} \\
d N_{x}=-\frac{x}{h p} \rho_{t} d s \\
d N_{y}=-\frac{y}{h p} \rho_{t} d s \\
\frac{d N_{x}}{d N_{y}}=\frac{x}{y}
\end{gathered}
$$

Donde:

xo: Punto inicial del desarrollo, 1er corte con el eje x-x (Condición de contorno).

FHT: Fuerza axil en el tablero en el primer punto del desarrollo (Positiva en el tablero a tracción y negativa en el tablero a compresión) (Condición de contorno).

$\rho_{\mathrm{T}}$ : Carga vertical por metro lineal de tablero (Condición de contorno).

hp: Altura de la torre sobre el tablero (Condición de contorno).

s: Variable del problema, longitud del tablero.

$\mathrm{x}$ : Coordenada «x» del tablero.

y: Coordenada «y» del tablero.

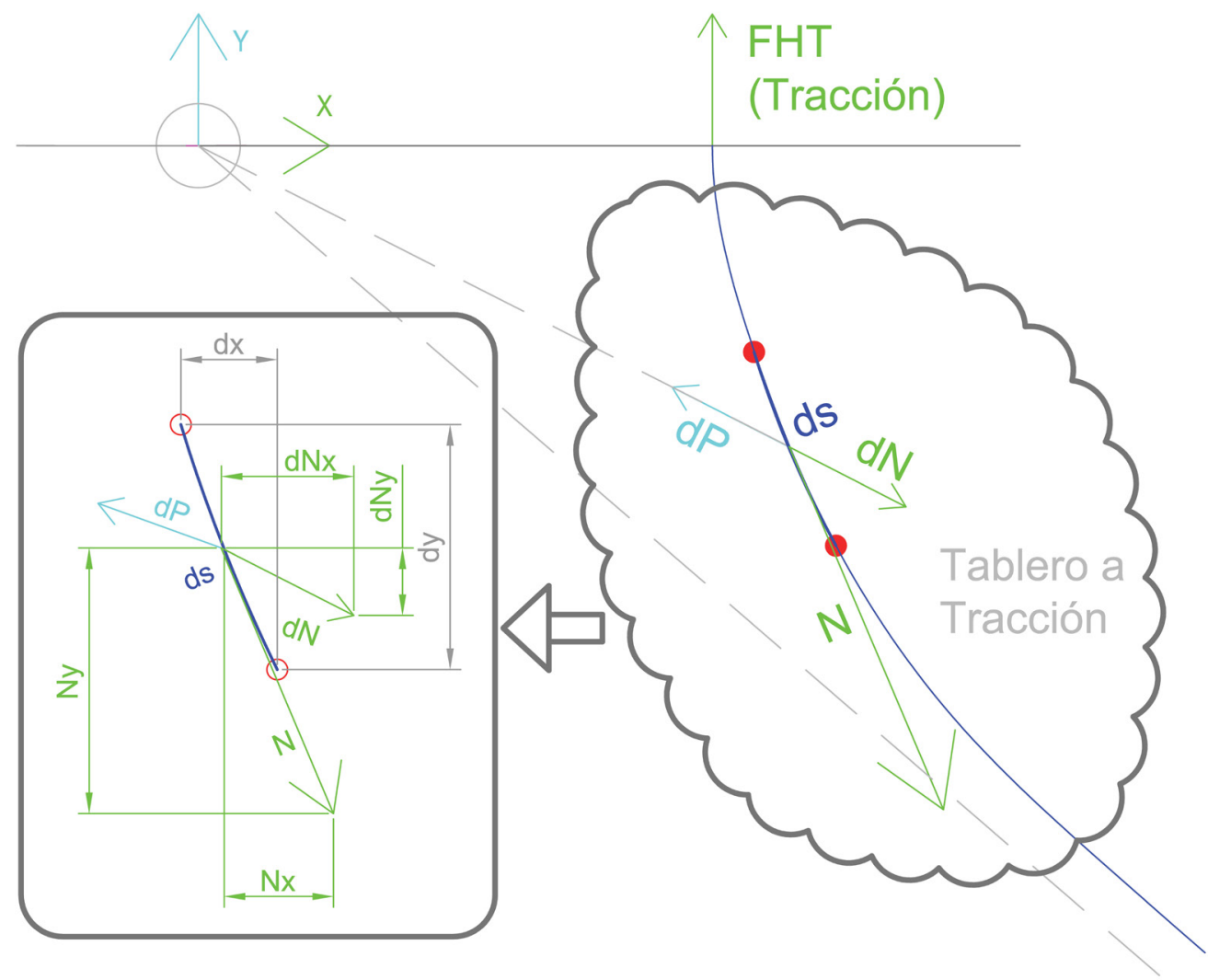

Figura 7. Cálculo continuo de directriz. Detalle diferencial de tablero. 
N: Fuerza directriz del trazado del tablero (coincide con la solicitación axil del tablero).

$\mathrm{Nx}$ : Componente en «X» de la fuerza directriz del trazado del tablero (coincide con la solicitación axil del tablero).

Ny: Componente en «y» de la fuerza directriz del trazado del tablero (coincide con la solicitación axil del tablero).

En la Figura 7, se observa la relación inmediata que existe entre la posición del tablero y su axil. De forma que el vector de la fuerza directriz $\mathrm{N}$ del tablero, influye de 2 formas sobre la directriz del tablero, en primer lugar, su dirección nos va a marcar en todo momento la dirección hacia la que se dirige el tablero, mientras que su módulo nos va a marcar lo estable que es esta dirección. Esta relación directa entre módulo y estabilidad de directriz (o lo que es lo mismo, una relación inversa entre curvatura y módulo del axil), va a ser muy importante de cara a la solicitación transversal que va a tener que sufrir el tablero debido a la discontinuidad que suponen los apoyos en los estribos. Lógicamente, cuanto más estable sea la directriz menor será esta solicitación transversal.

Se han supuesto, por simplicidad de cálculo, las mismas condiciones de contorno que en el cálculo discreto. Con esas condiciones de contorno, vamos a describir las ecuaciones que solucionan el problema.

Condiciones de contorno (2 primeros puntos).

Tablero a compresión y tablero a tracción

\begin{tabular}{|c|}
\hline$N_{x}[0]=0$ \\
\hline$N_{x}^{\prime}[0]=-x_{o} \frac{\rho_{T}}{h p}$ \\
\hline$N_{y}[0]=-F H T$ \\
\hline$N_{y}^{\prime}[0]=0$ \\
\hline
\end{tabular}

Tablero a compresión:

$$
\begin{aligned}
& \mathrm{N}_{\mathrm{x}}[\mathrm{s}]=\sqrt{\mathrm{N}_{\mathrm{x}}[\mathrm{s}]^{2}+\mathrm{N}_{\mathrm{y}}[\mathrm{s}]^{2}} \mathrm{~N}_{\mathrm{x}}[\mathrm{s}] \frac{\mathrm{hp}}{\rho_{\mathrm{T}}} \\
& \mathrm{N}_{\mathrm{y}}[\mathrm{s}]=\sqrt{\mathrm{N}_{\mathrm{x}}[\mathrm{s}]^{2}+\mathrm{N}_{\mathrm{y}}[\mathrm{s}]^{2}} \mathrm{~N}_{\mathrm{y}}^{\prime \prime}[\mathrm{s}] \frac{\mathrm{hp}}{\rho_{\mathrm{T}}}
\end{aligned}
$$

Tablero a Tracción:

$$
\begin{aligned}
& \mathrm{N}_{\mathrm{x}}[\mathrm{s}]=-\sqrt{\mathrm{N}_{\mathrm{x}}[\mathrm{s}]^{2}+\mathrm{N}_{\mathrm{y}}[\mathrm{s}]^{2}} \mathrm{~N}_{\mathrm{x}}[\mathrm{s}] \frac{\mathrm{hp}}{\rho_{\mathrm{T}}} \\
& \mathrm{N}_{\mathrm{y}}[\mathrm{s}]=-\sqrt{\mathrm{N}_{\mathrm{x}}[\mathrm{s}]^{2}+\mathrm{N}_{\mathrm{y}}[\mathrm{s}]^{2}} \mathrm{~N}_{\mathrm{y}}[\mathrm{s}] \frac{\mathrm{hp}}{\rho_{\mathrm{T}}}
\end{aligned}
$$

Donde los significados ya se han dado para las expresiones [11] a [16].

Se puede apreciar como en las ecuaciones (expresiones [18][21]) diferenciales sólo aparecen las funciones de fuerza $(\mathrm{Nx}[\mathrm{s}]$ y $\mathrm{Ny}[\mathrm{s}])$ [18]-[21], cuando realmente lo que queremos son las funciones de posición (x[s] e y[s]) que son las que nos van a dar la directriz del tablero. La razón está en la imposibilidad de despejar para dejar un sistema de ecuaciones diferenciales con las 2 funciones de posición como incógnitas.
Para obtener las funciones de posición, podemos:

a) Realizar un cambio de variable que nos permita despejar las funciones de fuerza, dejando exclusivamente las de posición.

Se hace un cambio de variable, similar a los que se realizan para pasar en dinámica, de la variable espacio [22], a la variable tiempo (21). Es por esta razón, por la que la nueva variable la hemos llamado $t_{\mathrm{T}}$.

$$
\mathrm{dt}_{\mathrm{T}}=\frac{\mathrm{ds}}{\mathrm{N}}
$$

Pero este método va a tener como grandes desventajas que " $\mathrm{t}_{\mathrm{T}}$ » tiene una relación con la variable que nos interesa («S») no lineal y que las funciones de posición se vuelven muy inestables, en el tablero a tracción, a partir de un determinado «t $\mathrm{t}_{\mathrm{T}}$ final». Recordemos que el incremento de la aceleración del sistema dinámico equivalente, depende linealmente de la distancia a la torre. Luego hay un punto en el que la velocidad crece descontroladamente por unidad de tiempo, haciendo el problema inmanejable.

b) Derivar las funciones de fuerza, obtenidas mediante sus funciones diferenciales (Compresión: [18] y [19], Tracción: [20] y [21]). Para obtener las de posición con las expresiones [23] y [24].

$$
\begin{aligned}
& x=-\frac{d N_{x}}{d s} \frac{h p}{\rho_{t}} \\
& y=-\frac{d N_{y}}{d s} \frac{h p}{\rho_{t}}
\end{aligned}
$$

La ventaja de esta vía, radica en que siempre vamos a tener las funciones dependientes de la variable esencial del problema «s», mientras que su desventaja está en que hay que diferenciar las funciones de fuerza axil del tablero para obtener la directriz. En el siguiente apartado se verá que la resolución numérica del sistema de ecuaciones diferenciales ([18]-[21]) de las fuerzas, nos va a dar directamente la solución de fuerzas y de coordenadas.

La conclusión es que la segunda vía es mucho más sencilla desde todos los puntos de vista. Luego es solo esta vía la que se va a desarrollar a partir de este punto.

\subsection{Solución de la ecuación diferencial}

El sistema de ecuaciones diferenciales de fuerzas en función de la variable «s», es no lineal de segundo orden. Su solución analítica es inabordable desde los métodos clásicos de resolución de sistemas de ecuaciones diferenciales, aunque es un sistema de ecuaciones diferenciales ordinario debido a su dependencia exclusiva de una variable. Por este motivo, se ha optado por una aproximación numérica mediante el método de Runge-Kutta. En la resolución, vamos a transformar el sistema de 2 ecuaciones de segundo orden, en uno de 4 ecuaciones de primer orden, con 2 nuevas variables, salidas de las expresiones [25] y [26].

$$
\begin{aligned}
& x=-\frac{d_{x}}{d s} \frac{h p}{\rho_{t}} \\
& y=-\frac{d N_{y}}{d s} \frac{h p}{\rho_{t}}
\end{aligned}
$$




\begin{tabular}{|c|}
\hline $\begin{array}{l}\text { Condiciones de contorno (2 primeros puntos). } \\
\text { Tablero a compresión y tablero a tracción }\end{array}$ \\
\hline $\mathrm{N}_{\mathrm{x}}[\mathrm{o}]=\mathrm{o}$ \\
\hline $\mathrm{x}[\mathrm{o}]=\mathrm{x}_{\mathrm{o}}$ \\
\hline $\mathrm{N}_{\mathrm{x}}^{\prime}[\mathrm{o}]=-\mathrm{x}_{\mathrm{o}} \frac{\rho_{\mathrm{T}}}{\mathrm{hp}}$ \\
\hline $\mathrm{x}^{\prime}[\mathrm{o}]=\mathrm{o}$ \\
\hline $\mathrm{N}_{\mathrm{y}}[\mathrm{o}]=-\mathrm{FHT}$ \\
\hline $\mathrm{y}^{\mathrm{y}}[\mathrm{o}]=\mathrm{o}$ \\
\hline $\mathrm{N}_{\mathrm{y}}^{\prime}[\mathrm{o}]=\mathrm{o}$ \\
\hline $\mathrm{y}^{\prime}[\mathrm{o}]=-1$ \\
\hline
\end{tabular}

Tablero a compresión queda definido por las expresiones [25], [26], [28] y [29].

$$
\begin{aligned}
& \mathrm{N}_{\mathrm{x}}[\mathrm{s}]=\sqrt{\mathrm{N}_{\mathrm{x}}[\mathrm{s}]^{2}+\mathrm{N}_{\mathrm{y}}[\mathrm{s}]^{2}} \mathrm{x}^{\prime}[\mathrm{s}] \\
& \mathrm{N}_{\mathrm{y}}[\mathrm{s}]=\sqrt{\mathrm{N}_{\mathrm{x}}[\mathrm{s}]^{2}+\mathrm{N}_{\mathrm{y}}[\mathrm{s}]^{2}} \mathrm{y}^{\prime}[\mathrm{s}]
\end{aligned}
$$

Tablero a tracción queda definido por las expresiones [25], [26], [30] y [31].

$$
\begin{aligned}
& N_{x}[s]=-\sqrt{N_{x}[s]^{2}+N_{y}[s]^{2}} x^{\prime}[s] \\
& N_{y}[s]=-\sqrt{N_{x}[s]^{2}+N_{y}[s]^{2}} y^{\prime}[s]
\end{aligned}
$$

Como se aprecia en la Figura 8, con el cálculo discreto y el cálculo continuo se obtienen directrices idénticas. Luego los análisis realizados para las curvas desarrolladas mediante el método discreto (Figuras 3 a 6) son válidos para esta metodología.

\section{CONCLUSIÓN SOBRE LOS CÁLCULOS REALIZADOS}

Se ha llegado a la conclusión de que la aproximación más realista viene por una resolución numérica del problema. Mediante un cálculo discreto, introduciendo nudos a una determinada distancia que nos permita reunir la información necesaria para obtener el siguiente o mediante la aproximación numérica a la solución de las ecuaciones diferenciales no lineales obtenidas a partir de un análisis continuo del problema, que no deja de ser otra discretización del problema.

En cualquier caso, una vez realizados distintos tableros y analizados los resultados, se ha comprobado que los resultados obtenidos de estas aproximaciones numéricas son, similares como muestra la Figura 8 y completamente satisfactorios frente a lo esperado bajo las hipótesis con las que se inició el problema.

\section{Tablero a Compresión}

\section{Directriz mediante cálculo contínuo}

\section{Directriz mediante cálculo discreto}

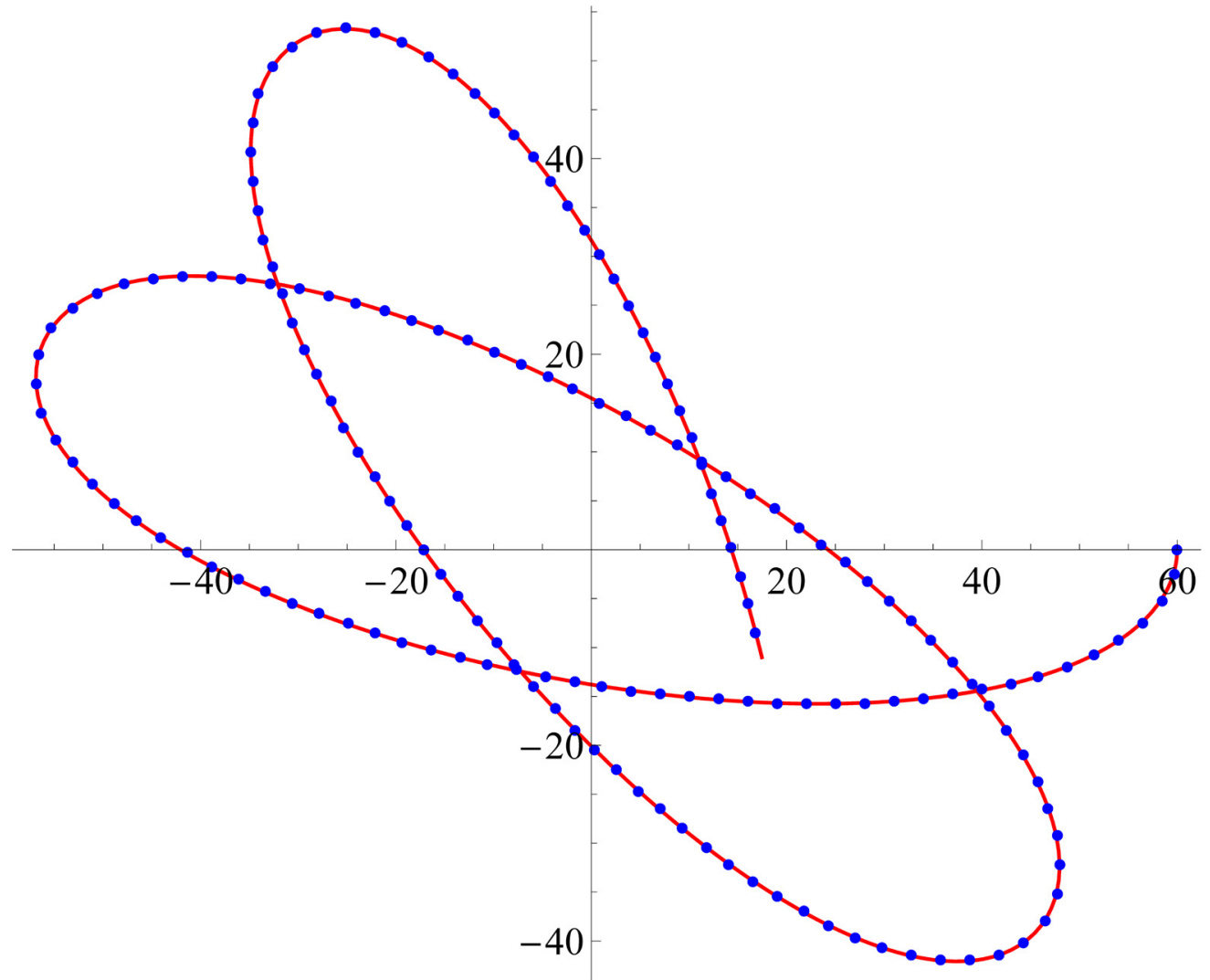

Figura 8. Comparación entre el cálculo discreto y continuo de la directriz del tablero. 
De cara a una implantación y posible uso de este tipo de trazados en el diseño real de puentes, se va a hacer una aproximación mediante mínimos cuadrados de las soluciones obtenidas numéricamente.

\section{APROXIMACIÓN POR MÍNIMOS CUADRADOS}

\subsection{Tablero a compresión}

La forma que más se aproxima a la directriz descrita por las ecuaciones diferenciales obtenidas, es la de un hipotrocoide. Para facilitar la aplicación de estos trazados, se ha realizado una aproximación mediante mínimos cuadrados. Con este fin se ha estudiado el trazado para advertir los puntos singulares que nos van a permitir caracterizar cada trazado según sean sus condiciones de contorno.

Tal y como se ha planteado el problema (expresión [27]), el trazado del tablero comienza $(\mathrm{s}=\mathrm{O})$ en un punto singular (en el caso desarrollado para la Figura 3 se trata de un máximo de radio y un mínimo de fuerza, como se observa en la Figura 4). Existen unas determinadas condiciones de contorno, para las que la figura desarrollada es un círculo (esto es obvio, si se tiene en cuenta que es el mecanismo de una rueda de bicicleta sigue este principio). En ese caso el radio y el axil se mantendrían constantes a lo largo del trazado. Para que tengamos un círculo, se tiene que cumplir la expresión [32].

$$
\mathrm{FHT}=\mathrm{x}_{\mathrm{o}}{ }^{2} \frac{\rho_{\mathrm{T}}}{\mathrm{hp}}
$$

Este valor de la fuerza en el inicio del tablero [32], es la frontera a partir de la cual cambiamos el tipo de punto singular desde el que comenzamos. Si FHT es menor que este valor, estaremos en un máximo de radio mínimo de fuerza. Si por el contrario es mayor, estaremos en un máximo de fuerza y mínimo de radio. A partir de punto se han estudiado las siguientes variables adimensionales, que marcan la directriz del tablero (Figura 3):

- $\operatorname{Abs}\left[\frac{\mathrm{FHT}}{\mathrm{x}_{\mathrm{o}}{ }^{2} \frac{\rho_{\mathrm{T}}}{\mathrm{hp}}}\right]$ : Relación entre la fuerza axil del tablero en el punto de inicio y la fuerza que se debe aplicar para que la directriz sea un círculo (en valor absoluto).

- $\operatorname{Abs}\left[\frac{\mathrm{N}_{\min }}{\mathrm{R}_{\max }{ }^{2} \frac{\rho_{\mathrm{T}}}{\mathrm{hp}}}\right]$ : Es la relación entre el mínimo axil del ta-

blero a lo largo de su directriz (que coincidirá con Rmax) y el axil que debería tener en ese mismo punto singular para que su directriz fuese una circunferencia de radio Rmax (en valor absoluto).

- $\frac{\mathrm{R}_{\min }}{\mathrm{x}_{\mathrm{o}}}$ : Relación entre el radio menor y radio mayor de la directriz.

- $\beta_{\min }$ : Ángulo cubierto entre puntos singulares.

Partimos de la primera variable $\operatorname{Abs}\left[\frac{\mathrm{FHT}}{\mathrm{x}_{\mathrm{o}}{ }^{2} \frac{\rho_{\mathrm{T}}}{\mathrm{hp}}}\right]$, que relaciona

todas las condiciones de contorno del problema. Si es menor que la unidad, del estudio de diferentes trazados de tableros a compresión, vemos que tanto $\frac{\mathrm{R}_{\min }}{\mathrm{x}_{\mathrm{o}}}$ como $\beta_{\min }$ son funciones dependientes exclusivamente de $\operatorname{Abs}\left[\frac{\mathrm{N}_{\min }}{\mathrm{R}_{\max }{ }^{2} \frac{\rho_{\mathrm{T}}}{\mathrm{hp}}}\right]$. Esto nos permite aproximar estas funciones adimensionales, mediante mínimos cuadrados, para obtener 2 ecuaciones más que resuelvan las incógnitas de las ecuaciones del hipotrocoide, definidas por las expresiones [33] y [34].

$$
\begin{aligned}
& x(t)=(a-b) \cos [t]+c \cos \left[\frac{a-b}{b} t\right] \\
& y(t)=(a-b) \sin [t]-c \sin \left[\frac{a-b}{b} t\right]
\end{aligned}
$$

Las expresiones [35], [36], [37], [38] y [39], nos solucionan las incógnitas.

$$
\begin{gathered}
\mathrm{R}_{\text {max }}=\mathrm{x}_{\mathrm{o}} \\
\mathrm{N}_{\text {min }}=\mathrm{FHT}
\end{gathered}
$$

Con los siguientes resultados de las expresiones [37], [38] y [39].

$$
\begin{gathered}
\mathrm{a}=\frac{\pi\left(\mathrm{R}_{\text {min }}-\mathrm{R}_{\text {max }}\right)}{2 \beta_{\text {min }}} \\
\mathrm{b}=\frac{\left(\pi+\beta_{\text {min }}\right)\left(\mathrm{R}_{\text {min }}-\mathrm{R}_{\text {max }}\right)}{2 \beta_{\text {min }}} \\
\mathrm{c}=\frac{\left(\mathrm{R}_{\text {min }}+\mathrm{R}_{\text {max }}\right)}{2}
\end{gathered}
$$

Y las aproximaciones mediante mínimos cuadrados se observan en las expresiones [40] y [41].

$$
\begin{aligned}
& \frac{\mathrm{R}_{\text {min }}}{\mathrm{R}_{\max }}=1.868674 \mathrm{Cf}-2.004082 \mathrm{Cf}^{2}+1.8051354 \mathrm{Cf}^{3}-0.6697273 \mathrm{Cf}^{4} \\
& \beta_{\text {int }}=\frac{-0.0008611-0.486716 \mathrm{Cf}-24.281 \mathrm{Cf}^{2}+192.151 \mathrm{Cf}^{3}-\mathrm{Cf}^{4}}{0.000548213+0.307257 \mathrm{Cf}+14.6197 \mathrm{Cf}^{2}+103.169 \mathrm{Cf}^{3}+\mathrm{Cf}^{4}}
\end{aligned}
$$

Con la variable definida en la expresión [42].

$$
\mathrm{Cf}=\mathrm{Abs}\left[\frac{\mathrm{N}_{\min }}{\mathrm{R}_{\max }{ }^{2} \frac{\rho_{\mathrm{T}}}{\mathrm{hp}}}\right]
$$

Si por el contrario $\operatorname{Abs}\left[\frac{\mathrm{FHT}}{\mathrm{x}_{\mathrm{o}}{ }^{2} \frac{\rho_{\mathrm{T}}}{\mathrm{hp}}}\right]>1$, estamos en un punto

de mínimo de radio y máximo de fuerza. Para no introducir nuevas ecuaciones aproximadas, sabiendo que las que se han mostrado para las diferentes incógnitas relacionan todas las variables necesarias. Se ha desarrollado una aproximación para la relación entre fuerzas en el máximo y mínimo de la directriz [43]. De esta manera nuestra incógnita pasa a ser Cf, a partir de la cual podemos obtener, en este caso, $\mathrm{R}_{\max } \mathrm{y} \beta_{\text {int }}$. 


$$
\mathrm{Cf}=\frac{101.35885+15.37035 \mathrm{Cfm}+0.100525 \mathrm{Cfm}^{2}}{-4.78159+117.93709 \mathrm{Cfm}+5.16712 \mathrm{Cfm}^{2}+0.0044895 \mathrm{Cfm}^{3}}
$$

Con:

$$
\begin{gathered}
\mathrm{Cfm}=\operatorname{Abs}\left[\frac{\mathrm{FHT}}{\mathrm{x}_{\mathrm{o}}{ }^{2} \frac{\rho_{\mathrm{T}}}{\mathrm{hp}}}\right] \\
\mathrm{R}_{\max }=\mathrm{x}_{\mathrm{o}} \\
\mathrm{N}_{\max }=\mathrm{FHT}
\end{gathered}
$$

La nueva directriz quedará definida por las expresiones [47] y [48], donde los valores de las incógnitas se siguen obteniendo de las expresiones [37], [38] y [39].

$$
\begin{aligned}
& x(t)=-(a-b) \cos [t]+c \cos \left[\frac{a-b}{b} t\right] \\
& y(t)=-(a-b) \sin [t]-c \sin \left[\frac{a-b}{b} t\right]
\end{aligned}
$$

\subsection{Tablero a tracción}

En el caso del tablero solicitado a tracción (funicular), se puede apreciar como tenemos una asíntota oblicua muy clara que pasa por el centro de coordenadas. Solo existe un punto singular, que coincide con el punto de inicio del trazado. A partir de la asíntota, se puede elaborar una parametrización de las curvas, de nuevo mediante aproximaciones mediante el método de los mínimos cuadrados.
La aproximación al problema va a ser diferente a la realizada para el tablero a compresión, en este caso la aproximación va a ser a una curva racional de cuarto grado. Aunque como ocurría en aquel, la expresión [32] permite obtener el resto de datos de la directriz del tablero. Mediante las siguientes expresiones adimensionales (Figura 5):

- $\mathrm{Cf}=\mathrm{Abs}\left[\frac{\mathrm{FHT}}{\mathrm{x}_{\mathrm{o}}{ }^{2} \frac{\rho_{\mathrm{T}}}{\mathrm{hp}}}\right]$ : Relación entre la fuerza axil del tablero en el punto de inicio y la fuerza que se debe aplicar para que la directriz sea un círculo, en el caso de un tablero a compresión. - $\beta_{\text {asin }}=\operatorname{Atan}\left[\lim _{\mathrm{s} \rightarrow \infty} \frac{\mathrm{N}_{\mathrm{y}}}{\mathrm{N}_{\mathrm{x}}}\right]$ : Es el ángulo de la asíntota oblicua que marca la directriz del tablero de tracción cuando «s» tiende a infinito.

- $\beta f=\frac{\beta}{\beta_{\text {asin }}}$ : Relación entre la coordenada angular de la directriz del tablero y el ángulo de la asíntota oblicua de la directriz del tablero cuando «S» tiende a infinito.

- $\mathrm{Rf}=\frac{\mathrm{R}}{\mathrm{x}_{\mathrm{o}}}$ : Relación entre la coordenada radial de la directriz del puente y la coordenada abscisa inicial.

Como se puede intuir de las variables adimensionales presentadas, el planteamiento de la aproximación se va a hacer en un sistema de coordenadas cilíndricas, porque las relaciones paramétricas encontradas en la curva se simplifican mucho. La transformación a coordenadas ortogonales (en caso de ser necesario) es sencilla.

\section{Tablero a COMPRESIÓN}

$$
\begin{aligned}
& \operatorname{Abs}\left[\frac{\mathrm{FHT}}{\left(\mathrm{xo}^{2} \mathrm{Pv} / \mathrm{hp}\right)}\right]=\frac{25}{72} \\
& (\mathrm{xo}=120 \mathrm{~m}, \rho \mathrm{t}=-4 \mathrm{KN} / \mathrm{m}, \mathrm{hp}=40 \mathrm{~m}, \mathrm{FHT}=-500 \mathrm{KN})
\end{aligned}
$$

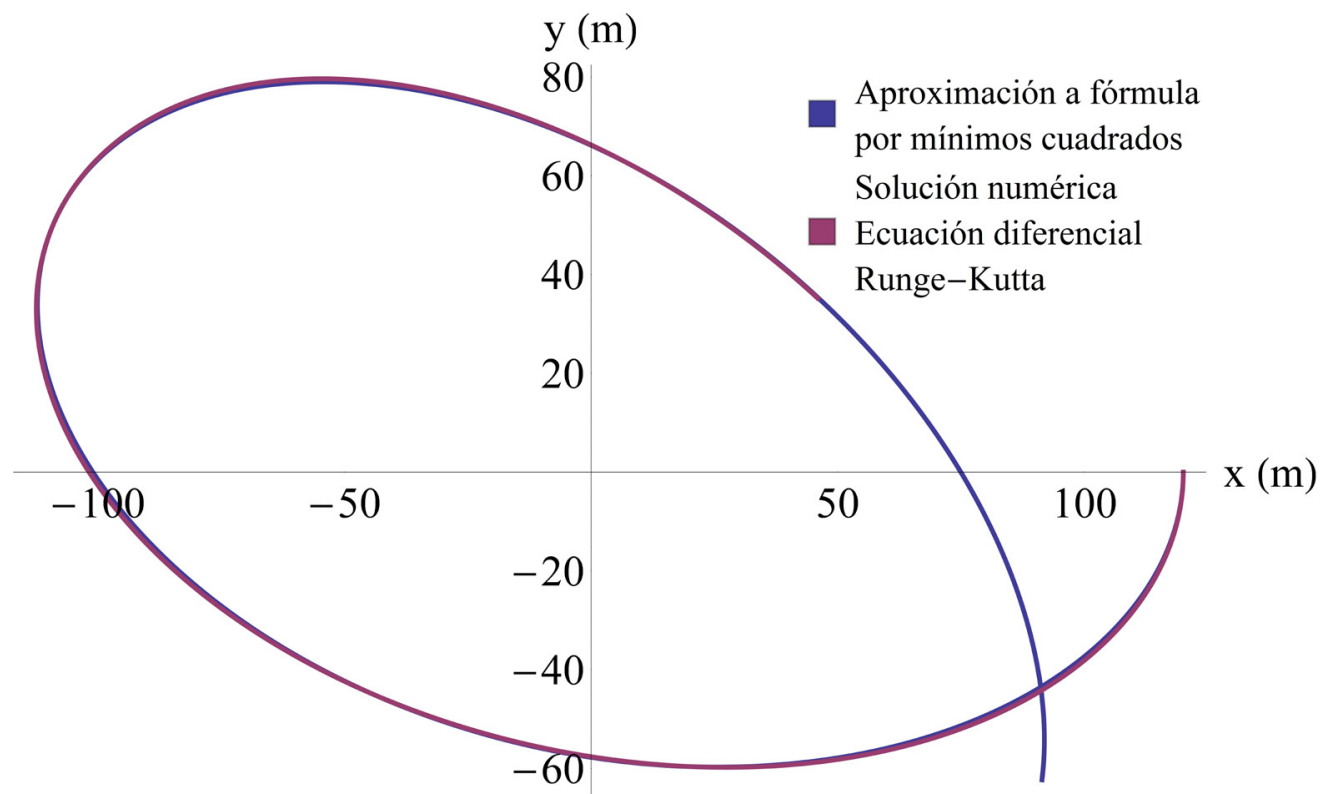

Figura 9. Aproximación a hipotrocoide directriz de tablero a compresión mediante mínimos cuadrados. 
Como en el caso del tablero a compresión, partimos de la primera variable $\operatorname{Abs}\left[\frac{\mathrm{FHT}}{\mathrm{x}_{\mathrm{o}}{ }^{2} \frac{\rho_{\mathrm{T}}}{\mathrm{hp}}}\right]$, que relaciona todas las condiciones de contorno del problema. Del estudio de las diferentes curvas que se dan en los tableros a tracción, se ha concluido que la asíntota oblicua $\beta_{a \sin }=\operatorname{Atan}\left[\lim _{\mathrm{s} \rightarrow \infty} \frac{\mathrm{N}_{\mathrm{y}}}{\mathrm{N}_{\mathrm{x}}}\right]$, es función exclusivamente de $\mathrm{Cf}=\mathrm{Abs}\left[\frac{\mathrm{FHT}}{\mathrm{x}_{\mathrm{o}}{ }^{2} \frac{\rho_{\mathrm{T}}}{\mathrm{hp}}}\right]$. Mediante una aproximación de mínimos cuadrados, se ha llegado a la siguiente ecuación racional:

$$
\beta_{\text {asin }}=\frac{-126.511 \mathrm{Cf}-656.321 \mathrm{Cf}^{2}+155.665 \mathrm{Cf}^{3}-\frac{\pi}{2} \mathrm{Cf}^{4}}{32.7+514.791 \mathrm{Cf}+672.957 \mathrm{Cf}^{2}+111.067 \mathrm{Cf}^{3}+\mathrm{Cf}^{4}}
$$

Este ángulo de la asíntota, nos permite hacer adimensional la coordenada angular del problema: $\frac{\beta}{\beta_{\text {asin }}}$. De nuevo a partir de los valores obtenidos de las ecuaciones diferenciales, se concluye que la variable adimensional $\frac{\beta}{\beta_{\text {asin }}}$ obtenida a partir de la coordenada angular, solo es función de $\frac{R}{x_{0}} y$ viceversa. De nuevo mediante mínimos cuadrados, se obtiene las expresiones [50] y [51].

$\beta=\beta_{\text {asin }} \frac{-0.677747+1.28168 R f+0.46836 R f^{2}-2.07229 R f^{3}+R f^{4}}{-0.13952+0.09436 R f+1.20776 R f^{2}-2.16259 R f^{3}+R f^{4}}$ Con $R f \geq 1 \quad[50]$

a) Tablero a TRACCIÓN $(\beta)$

$$
\begin{aligned}
& \operatorname{Abs}\left[\frac{\mathrm{FHT}}{\left(\mathrm{xo}^{2} \mathrm{Pv} / \mathrm{hp}\right)}\right]=\frac{6}{5} \\
& (\mathrm{xo}=50 \mathrm{~m}, \rho \mathrm{t}=-4 \mathrm{KN} / \mathrm{m}, \mathrm{hp}=40 \mathrm{~m}, \mathrm{FHT}=300 \mathrm{KN})
\end{aligned}
$$

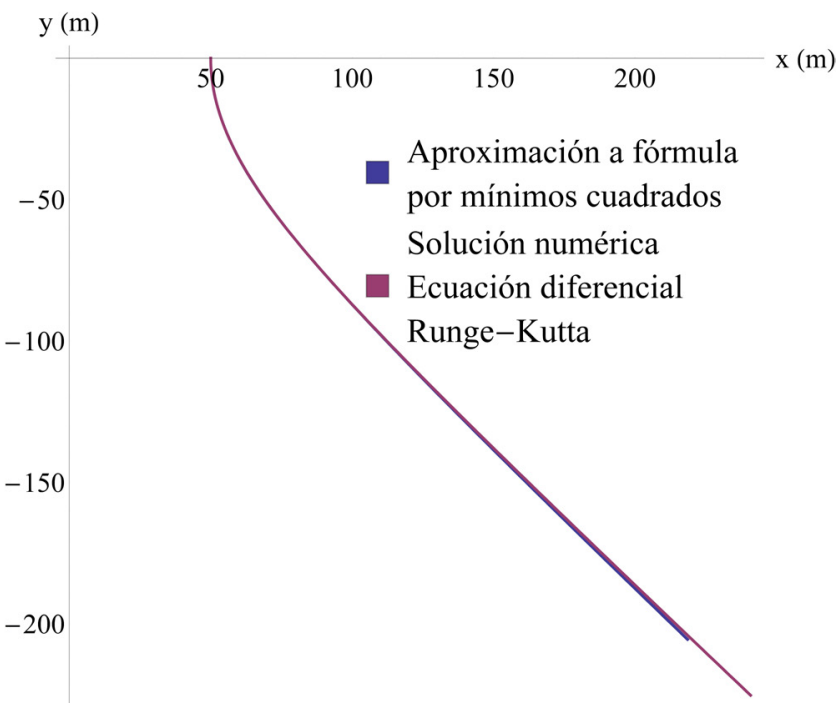

$R=x_{0} \frac{1-1.76725 \beta f+0.64759 \beta f^{2}+017558 \beta f^{3}+0.05433 \beta f^{4}}{1-1.76726 \beta f+0.17938 \beta f^{2}+1.00042 \beta f^{3}+0.41253 \beta f^{4}}$ Con $0 \leq \beta f \leq 1$

En la Figura 10, se muestran las aproximaciones conseguidas mediante las expresiones [50] y [51].

\section{EJEMPLOS}

Como punto final al artículo, se introducen 2 ejemplos de directrices tipo «Tablero-AXIL», uno a tracción y otro a compresión, para una pasarela peatonal de $110 \mathrm{~m}$ de longitud total y una anchura de $3 \mathrm{~m}$ (Figura 11). La sección del tablero es un cajón metálico, con un peso por metro lineal . En los ejemplos se puede apreciar la resultante de las cargas que introducen los tirantes en la cabeza de la torre (es obvio, que una torre que siguiese esa trayectoria evitaría acciones transversales).

\section{CONCLUSIÓN}

Se ha estudiado la posibilidad de optimizar los trazados de los puentes curvos atirantados eliminando las solicitaciones más perjudiciales de éstos, las transversales. Se ha planteado la solución del problema de forma discreta, mediante sumatorios, y de forma continua mediante un sistema de ecuaciones diferenciales. Las ecuaciones diferenciales se han resuelto numéricamente, por la imposibilidad de una solución analítica. Por último, se han aproximado los trazados, tanto para aquellos tableros que soportarían compresiones (curva antifunicular) como para aquellos que soportarían tracciones (curva funicular).

Este planteamiento abre un nuevo punto de vista a la hora de enfrentarse a un tablero curvo en el que no se van a poder realizar apoyos puntuales múltiples directos al terreno. La eliminación de las solicitaciones transversales para aquellas cargas que se considere oportuno, así como la posibilidad de

\section{b) Tablero a TRACCIÓN (R)}

$$
\begin{aligned}
& \operatorname{Abs}\left[\frac{\mathrm{FHT}}{\left(\mathrm{xo}^{2} \mathrm{Pv} / \mathrm{hp}\right)}\right]=\frac{6}{5} \\
& (\mathrm{xo}=50 \mathrm{~m}, \rho \mathrm{t}=-4 \mathrm{KN} / \mathrm{m}, \mathrm{hp}=40 \mathrm{~m}, \mathrm{FHT}=300 \mathrm{KN})
\end{aligned}
$$

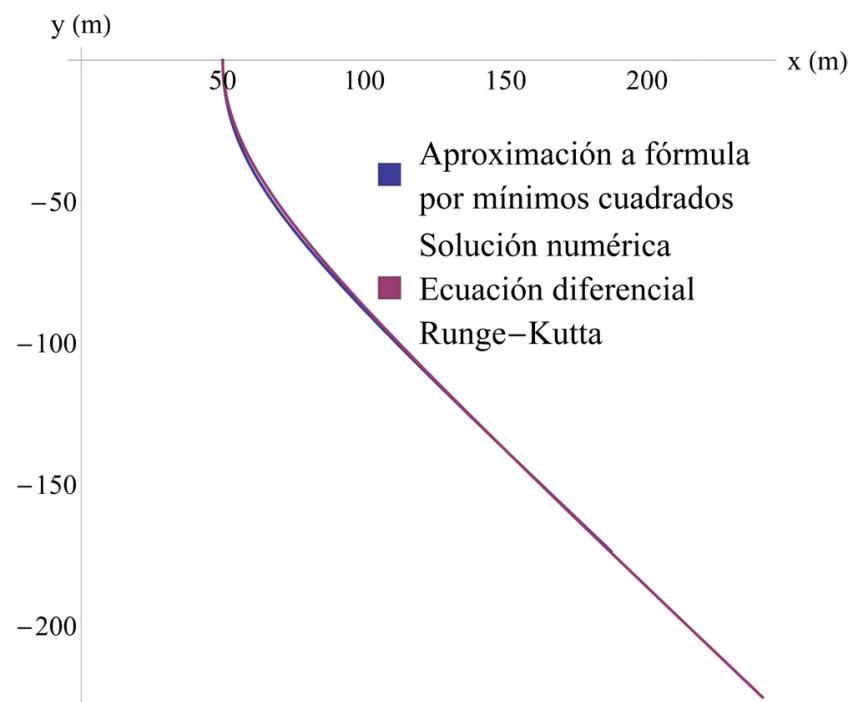

Figura 10. Aproximación a hipotrocoide directriz de tablero a tracción mediante mínimos cuadrados: a) Aproximando $\beta$, expresión [54]. a) Aproximando R, expresión [55]. 
a) Tablero (Compresión): FHT0 $=-770 \mathrm{KN}, \mathrm{xo}=25 \mathrm{~m}$, Ltablero $=110 \mathrm{~m}$. Alturas: pila hp $0=55, \mathrm{hp}(\mathrm{hp0}-\mathrm{ht})=35 \mathrm{~m}$, tablero $\mathrm{ht}=20 \mathrm{~m}$.

Xest $1=7.2951 \mathrm{~m}$, Yest $1=50.5936 \mathrm{~m}$, Xest $2=7.2951 \mathrm{~m}$, Yest $2=-50.5936 \mathrm{~m}$ Fest $1=457.61 \mathrm{KN}$, Fest $2=457.61 \mathrm{KN}$.

Pila inclinada al CDG

Nudos Tablero $n=56$, Tirantes $(n t-2)=10$.

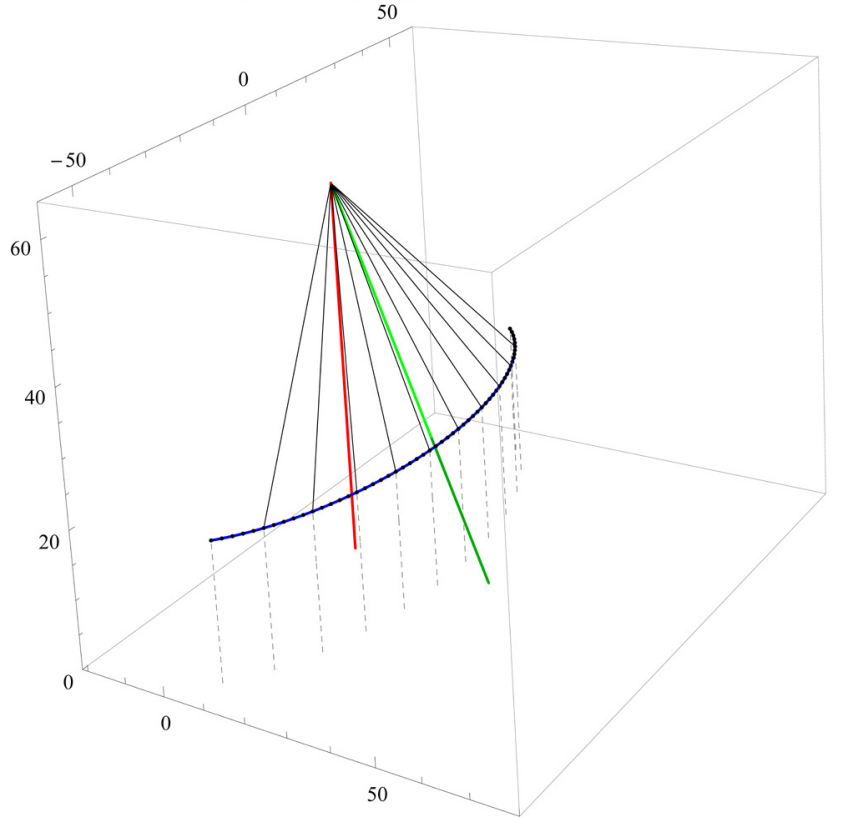

b) Tablero (TRACCIÓN): FHT0 $=132 \mathrm{KN}, \mathrm{xO}=10 \mathrm{~m}$, Ltablero $=110 \mathrm{~m}$. Alturas: pila $\mathrm{hp} 0=55, \mathrm{hp}(\mathrm{hp} 0-\mathrm{ht})=35 \mathrm{~m}$, tablero $\mathrm{ht}=20 \mathrm{~m}$.

Xest $1=28.9505 \mathrm{~m}$, Yest $1=51.1138 \mathrm{~m}$, Xest $2=28.9505 \mathrm{~m}$, Yest $2=-51.1138 \mathrm{~m}$ Fest $1=658.546 \mathrm{KN}$, Fest $2=658.546 \mathrm{KN}$

Pila inclinada al CDG

Nudos Tablero $n=56$, Tirantes $(n t-2)=10$.

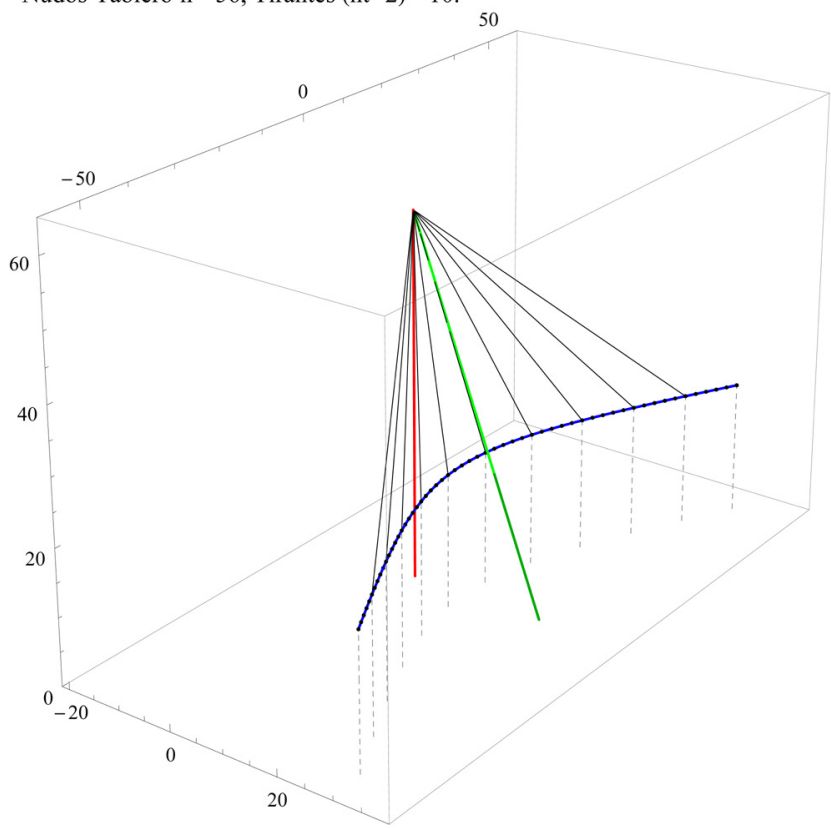

Figura 11. a) Tablero a compresión. b) Tablero a tracción.

modificar el trazado variando las condiciones de contorno, aportan al diseñador del puente una gran libertad. También es importante desde el punto de vista económico, sumándose la posibilidad de situar los diferentes elementos (esencial- mente estribos y torre) donde sean más efectivos y unas solicitaciones más beneficiosas sobre el tablero. A partir del estudio de la curvatura de la directriz, se abre la puerta de este tipo de diseños a posibles puentes atirantados para carretera.

\section{REFERENCIAS}

(1) Cagri Ozgur (2007). Behavior and analysis of a horizontally curved and skewed I-girder bridge (Tesis doctoral no publicada). Georgia: Georgia Institute of Technology.

(2) American Association of State Highway and Transportation Officials (2003). Guide Specifications for Horizontal Curved Steel Girder Highway Bridges (2003). Washington, DC (USA): (AASHTO).

(3) Rey-Rey, J. (2014). Aplicación de algoritmos paramétricos en el diseño estructural. La Ópera de Sídney desde una perspectiva contemporánea. Informes de la Construcción, 66(extra-1): mo16, doi: http://dx.doi.org/10.3989/ic.13.093.

(4) Avelino Samartín (1998). Una prospectiva de las tecnologías de las estructuras espaciales. Los puentes. Informes de la Construcción, 5O(455): 53-63, doi: http://dx.doi.org/10.3989/ic.1998.v50.i255.903

(5) Ursula Baus, Mike Schlaich (2007). Footbridges. Basel (Swizerland): Birkhauser Verlag.

(6) Lorenz Lachauer, Toni Kotnik (2011). Curved Bridge Design. En Gengnagel, C., Kilian, A., Palz, N., Scheurer, F. (Eds.), Computational Design Modelling (pp.145-152). Berlin (Germany): Springer. doi: http://dx.doi.org/10.1007/978-3-64223435-4_17

(7) Leonardo Todisco (2015). An integrated approach to conceptual design of arch bridges with curved deck (Tesis doctoral no publicada). Madrid (Spain): Escuela Técnica Superior de Caminos, Canales y Puertos.

(8) Rabasco, P. (2011). El sistema Ctesiphonte. Evolución de la estructura catenaria. Informes de la Construcción, 63(522): 43-52, doi: http://dx.doi.org/10.3989/ic.10.009.

(9) Heino Engel (2002). Sistemas de Estructuras. Barcelona: Editorial Gustavo Gili, SA.

(10) Jorge Maiztegui, Juan P. Durruty, Asdrúbal Bottano (2008). Vigas de eje curvo. Buenos Aires (Argentina): Facultad de Ingeniería Universidad Nacional de la Plata.

(11) Javier Manterola (2006). Puentes II. Madrid: Colegio de Ingenieros de Caminos Canales y Puertos, Colección Escuelas.

(12) Javier Manterola (2006). Puentes I. Madrid: Colegio de Ingenieros de Caminos Canales y Puertos, Colección Escuelas.

(13) Holger Svensson (2012). Cable-Stayed Bridges, 40 Years of Experience Worlwide. Düsseldorf (Germany): Helga Rutzen.

(14) Niels J. Gimsing, Christos T. Georgakis (2012). Cable Supported Bridges: Concept and Design. West Sussex (United Kingdom): John Wiley \& Sons Ltd.

(15) Darío Galante Bardín (2009). Diseño de una pasarela peatonal de $80 \mathrm{~m}$, basándose en los principios estructurales expuestos por Robert Le Ricolais. Madrid: Escuela Técnica Superior de Caminos, Canales y Puertos.

(16) Antonio González Serrano, Julio Besiga Díaz Blanco, Enrique Mitchel Esclusa (2004). Pasarela atirantada «LA ROSA» en la Coruña. Revista de obras públicas, 151 (3443): 31-39. 
(17) Javier Manterola Armisén, Miguel A. Gil Ginés, Miguel A. Astiz Suárez y Antonio Martinez Cutillas. Pasarelas. Revista de obras públicas, 146 (3384): 17-32.

(18) Jiri Strasky (2005). Stress ribbon and cable-supported pedestrian bridges. London (United Kingdom): Thomas Telford.

(19) René Walther (2007). Cable stayed bridges. London (United Kingdom): Thomas Telford.

(20) Miguel A. Astiz, Javier Manterola, Miguel A. Gil (2003). Pasarela atirantada sobre el Manzanares en Madrid. Informes de la Construcción, 55(485): 5-12, doi: http://dx.doi.org/10.3989/ic.2003.v55.i485.558.

(21) Sherif El-Tawil, Ayman M. Okeil (2006). Behavior and design of curved composite box girder bridges. Orlando: Department of Civil and Envioremental Engineering University of Central Florida.

(22) Cañete-Güeto, M., Fernández-Ruiz, M. A., Hernández-Montes, E. (2014). Método de Aproximación Catenaria en el diseño de estructuras antifuniculares. Informes de la Construcción, 66(extra-1): moo8, doi: http://dx.doi.org/10.3989/ ic.13.115.

(23) Ministerio de Fomento (1999). Trazado, Instrucción de Carreteras, Norma 3.1-IC. Madrid: Ministerio de Fomento. 